\title{
The MLE of the Parameters of a Discrete Competitive System Subject to Environmental Noise
}

\author{
Huili Xiang ${ }^{1,2}$ and Zhijun Liu ${ }^{1,2}$ \\ ${ }^{1}$ Key Laboratory of Biologic Resources Protection and Utilization of Hubei Province, Hubei University for Nationalities, \\ Enshi, Hubei 445000, China \\ ${ }^{2}$ Department of Mathematics, Hubei University for Nationalities, Enshi, Hubei 445000, China
}

Correspondence should be addressed to Zhijun Liu; zhijun_liu47@hotmail.com

Received 25 August 2014; Accepted 12 February 2015

Academic Editor: Josef Diblík

Copyright (C) 2015 H. Xiang and Z. Liu. This is an open access article distributed under the Creative Commons Attribution License, which permits unrestricted use, distribution, and reproduction in any medium, provided the original work is properly cited.

\begin{abstract}
A randomized discrete competitive system is investigated and the maximum likelihood estimation (MLE) of the parameters of the system is obtained. Also, a corresponding numerical simulation is offered to support our theoretical results.
\end{abstract}

\section{Introduction}

It is well known that various discrete competitive systems have received great attention owing to their theoretical and practical significance and there is a large volume of literature relevant to many good results (see [1-6]). Note that the population systems, in the real world, are often perturbed by various types of environmental noises. May [7] also pointed out that due to environmental fluctuation, the birth rate, the death rate, and other parameters usually show random fluctuation to a certain extent. To accurately describe such systems, it is necessary to use stochastic difference equations. In the present contribution, we will consider the maximum likelihood estimation (MLE) of the parameters of a discrete competitive system with environmental noise and our motivation comes from the works in [8-10]. Let us first introduce the following competitive system governed by differential equations:

$$
\begin{aligned}
& x_{1}^{\prime}(t)=x_{1}(t)\left(b_{1}-a_{11} x_{1}(t)-\frac{c_{2} x_{2}(t)}{1+x_{2}(t)}\right), \\
& x_{2}^{\prime}(t)=x_{2}(t)\left(b_{2}-a_{22} x_{2}(t)-\frac{c_{1} x_{1}(t)}{1+x_{1}(t)}\right),
\end{aligned}
$$

where $b_{i}, i=1,2$, represents the intrinsic growth rate; $a_{i i}$ and $c_{i}, i=1,2$, stand for intraspecific competing rate and interspecific competing rate, respectively. For the relevant ecology of model (1) we refer to the readers to [11]. Obviously, by a computation, system (1) becomes

$$
\begin{aligned}
& \frac{d \log x_{1}(t)}{d t}=b_{1}-a_{11} x_{1}(t)-\frac{c_{2} x_{2}(t)}{1+x_{2}(t)}, \\
& \frac{d \log x_{2}(t)}{d t}=b_{2}-a_{22} x_{2}(t)-\frac{c_{1} x_{1}(t)}{1+x_{1}(t)} .
\end{aligned}
$$

Considering system (2) with the equidistant points in time $0, \Delta t, 2 \Delta t, \ldots, n \Delta t$ for some $\Delta t>0$, we obtain

$$
\begin{aligned}
& \log x_{1, k}=\log x_{1, k-1}+\left(b_{1}-a_{11} x_{1, k-1}-\frac{c_{2} x_{2, k-1}}{1+x_{2, k-1}}\right) \Delta t, \\
& \log x_{2, k}=\log x_{2, k-1}+\left(b_{2}-a_{22} x_{2, k-1}-\frac{c_{1} x_{1, k-1}}{1+x_{1, k-1}}\right) \Delta t .
\end{aligned}
$$

Suppose that the parameter $b_{i}, i=1,2$, is stochastically perturbed in the following way:

$$
b_{i} \longrightarrow b_{i}+\sigma_{i} \varepsilon_{i, k}
$$


where $\varepsilon_{i, k} \sim N(0,1)$ and $\sigma_{i}^{2}$ stands for the noise intensity. Then system (3) can be described by the randomized equations:

$$
\begin{aligned}
\log x_{1, k}= & \log x_{1, k-1}+\left(b_{1}-a_{11} x_{1, k-1}-\frac{c_{2} x_{2, k-1}}{1+x_{2, k-1}}\right) \Delta t \\
& +\sigma_{1} \sqrt{\Delta} t \varepsilon_{1, k}, \\
\log x_{2, k}= & \log x_{2, k-1}+\left(b_{2}-a_{22} x_{2, k-1}-\frac{c_{1} x_{1, k-1}}{1+x_{1, k-1}}\right) \Delta t \\
& +\sigma_{2} \sqrt{\Delta} t \varepsilon_{2, k} .
\end{aligned}
$$

Here we choose initial value $x(0)=\left(x_{1}(0), x_{2}(0)\right) \in R_{+}^{2}$.

The main aim of this paper is to investigate the MLE of the parameters of system (5). To the best of our knowledge, there are few published papers concerned with system (5). The rest of this paper is organized as follows. Section 2 focuses on the maximum likelihood estimation of the parameters of system (5). Numerical simulation and discussion are presented in Section 3.

\section{The MLE of the Parameters}

In this section, we focus on the MLE of the parameters of system (5). Suppose that $\left\{\left(x_{1, k}, x_{2, k}\right), k=1,2, \ldots, n\right\}$ are the real observed values from system (5). For the sake of simplicity, let $u_{i, k}=\log x_{i, k}(i=1,2 ; k=1,2, \ldots, n)$. Suppose that $\left(U_{1,0}, U_{2,0}\right),\left(U_{1,1}, U_{2,1}\right), \ldots,\left(U_{1, n}, U_{2, n}\right)$ are the corresponding observed quantity and $\rho$ is the correlation coefficient of $U_{1, l}$ and $U_{2, l}, l=1,2, \ldots, n$. Then the following notations are used throughout this paper:

$$
\begin{gathered}
\alpha_{1}=\frac{\sigma_{2} \Delta t}{n}\left[\sum_{k=1}^{n} \exp \left(u_{1, k-1}\right)\right]^{2}-\sigma_{2} \Delta t \sum_{k=1}^{n} \exp \left(2 u_{1, k-1}\right), \\
\alpha_{2}=\frac{\sigma_{2} \Delta t}{n} \sum_{k=1}^{n} \exp \left(u_{1, k-1}\right) \sum_{k=1}^{n} \frac{\exp \left(u_{2, k-1}\right)}{1+\exp \left(u_{2, k-1}\right)} \\
-\sigma_{2} \Delta t \sum_{k=1}^{n} \frac{\exp \left(u_{1, k-1}+u_{2, k-1}\right)}{1+\exp \left(u_{2, k-1}\right)}, \\
\alpha_{3}=\rho \sigma_{1} \Delta t \sum_{k=1}^{n} \frac{\exp \left(u_{2, k-1}\right)}{1+\exp \left(u_{2, k-1}\right)} \\
-\frac{\rho \sigma_{1} \Delta t}{n} \sum_{k=1}^{n} \exp \left(u_{1, k-1}\right) \sum_{k=1}^{n} \frac{\exp \left(u_{1, k-1}\right)}{1+\exp \left(u_{1, k-1}\right)}, \\
\alpha_{4}=\rho \sigma_{1} \Delta t \sum_{k=1}^{n} \exp \left(u_{1, k-1}+u_{2, k-1}\right) \\
-\frac{\rho \sigma_{1} \Delta t}{n} \sum_{k=1}^{n} \exp \left(u_{1, k-1}\right) \sum_{k=1}^{n} \exp \left(u_{2, k-1}\right), \\
\beta_{1}=\frac{\sigma_{2} \Delta t}{n} \sum_{k=1}^{n} \frac{\exp \left(u_{2, k-1}\right)}{1+\exp \left(u_{2, k-1}\right)} \sum_{k=1}^{n} \exp \left(u_{1, k}\right) \\
-\sigma_{2} \Delta t \sum_{k=1}^{n} \frac{\exp \left(u_{1, k-1}+u_{2, k-1}\right)}{1+\exp \left(u_{2, k-1}\right)},
\end{gathered}
$$

$$
\begin{aligned}
\beta_{2}= & \frac{\sigma_{2} \Delta t}{n}\left[\sum_{k=1}^{n} \frac{\exp \left(u_{2, k-1}\right)}{1+\exp \left(u_{2, k-1}\right)}\right]^{2} \\
& -\sigma_{2} \Delta t \sum_{k=1}^{n} \frac{\exp \left(2 u_{2, k-1}\right)}{\left[1+\exp \left(u_{2, k-1}\right)\right]^{2}},
\end{aligned}
$$$$
\beta_{3}=\rho \sigma_{1} \Delta t \sum_{k=1}^{n} \frac{\exp \left(u_{1, k-1}+u_{2, k-1}\right)}{\left(1+\exp \left(u_{1, k-1}\right)\right)\left(1+\exp \left(u_{2, k-1}\right)\right)}
$$$$
-\frac{\rho \sigma_{1} \Delta t}{n} \sum_{k=1}^{n} \frac{\exp \left(u_{1, k-1}\right)}{1+\exp \left(u_{1, k-1}\right)} \sum_{k=1}^{n} \frac{\exp \left(u_{2, k-1}\right)}{1+\exp \left(u_{2, k-1}\right)},
$$$$
\beta_{4}=\rho \sigma_{1} \Delta t \sum_{k=1}^{n} \frac{\exp \left(2 u_{2, k-1}\right)}{1+\exp \left(u_{2, k-1}\right)}
$$$$
-\frac{\rho \sigma_{1} \Delta t}{n} \sum_{k=1}^{n} \frac{\exp \left(u_{2, k-1}\right)}{1+\exp \left(u_{2, k-1}\right)} \sum_{k=1}^{n} \exp \left(u_{2, k-1}\right) \text {, }
$$$$
\gamma_{1}=\rho \sigma_{2} \Delta t \sum_{k=1}^{n} \frac{\exp \left(2 u_{1, k-1}\right)}{1+\exp \left(u_{1, k-1}\right)}
$$$$
-\frac{\rho \sigma_{2} \Delta t}{n} \sum_{k=1}^{n} \frac{\exp \left(u_{1, k-1}\right)}{1+\exp \left(u_{1, k-1}\right)} \sum_{k=1}^{n} \exp \left(u_{1, k-1}\right),
$$$$
\gamma_{2}=\rho \sigma_{2} \Delta t \sum_{k=1}^{n} \frac{\exp \left(u_{1, k-1}+u_{2, k-1}\right)}{\left(1+\exp \left(u_{1, k-1}\right)\right)\left(1+\exp \left(u_{2, k-1}\right)\right)}
$$$$
-\frac{\rho \sigma_{2} \Delta t}{n} \sum_{k=1}^{n} \frac{\exp \left(u_{1, k-1}\right)}{1+\exp \left(u_{1, k-1}\right)} \sum_{k=1}^{n} \frac{\exp \left(u_{2, k-1}\right)}{1+\exp \left(u_{2, k-1}\right)},
$$$$
\gamma_{3}=\frac{\sigma_{1} \Delta t}{n}\left[\sum_{k=1}^{n} \frac{\exp \left(u_{1, k-1}\right)}{1+\exp \left(u_{1, k-1}\right)}\right]^{2}
$$$$
-\sigma_{1} \Delta t \sum_{k=1}^{n} \frac{\exp \left(2 u_{1, k-1}\right)}{\left[1+\exp \left(u_{1, k-1}\right)\right]^{2}} \text {, }
$$$$
\gamma_{4}=\frac{\sigma_{1} \Delta t}{n} \sum_{k=1}^{n} \frac{\exp \left(u_{1, k-1}\right)}{1+\exp \left(u_{1, k-1}\right)} \sum_{k=1}^{n} \exp \left(u_{2, k}\right)
$$$$
-\sigma_{1} \Delta t \sum_{k=1}^{n} \frac{\exp \left(u_{1, k-1}+u_{2, k-1}\right)}{1+\exp \left(u_{1, k-1}\right)}
$$

$$
\begin{aligned}
\eta_{1}= & \rho \sigma_{2} \Delta t \sum_{k=1}^{n} \exp \left(u_{1, k-1}+u_{2, k-1}\right) \\
& -\frac{\rho \sigma_{2} \Delta t}{n} \sum_{k=1}^{n} \exp \left(u_{1, k-1}\right) \sum_{k=1}^{n} \exp \left(u_{2, k-1}\right),
\end{aligned}
$$$$
\eta_{2}=\rho \sigma_{2} \Delta t \sum_{k=1}^{n} \frac{\exp \left(2 u_{2, k-1}\right)}{1+\exp \left(u_{2, k-1}\right)}
$$$$
-\frac{\rho \sigma_{2} \Delta t}{n} \sum_{k=1}^{n} \exp \left(u_{2, k-1}\right) \sum_{k=1}^{n} \frac{\exp \left(u_{2, k-1}\right)}{1+\exp \left(u_{2, k-1}\right)},
$$ 


$$
\begin{aligned}
& \eta_{3}=\frac{\sigma_{1} \Delta t}{n} \sum_{k=1}^{n} \exp \left(u_{2, k-1}\right) \sum_{k=1}^{n} \frac{\exp \left(u_{1, k-1}\right)}{1+\exp \left(u_{1, k-1}\right)} \\
& -\sigma_{1} \Delta t \sum_{k=1}^{n} \frac{\exp \left(u_{1, k-1}+u_{2, k-1}\right)}{1+\exp \left(u_{1, k-1}\right)} \\
& \eta_{4}=\frac{\sigma_{1} \Delta t}{n}\left[\sum_{k=1}^{n} \exp \left(u_{2, k-1}\right)\right]^{2}-\sigma_{1} \Delta t \sum_{k=1}^{n} \exp \left(2 u_{2, k-1}\right) \text {, } \\
& \zeta_{1}=\sigma_{2} \sum_{k=1}^{n}\left(u_{1, k}-u_{1, k-1}\right) \exp \left(u_{1, k-1}\right) \\
& -\frac{\sigma_{2}\left(u_{1, n}-u_{1,0}\right)}{n} \sum_{k=1}^{n} \exp \left(u_{1, k-1}\right) \\
& +\frac{\rho \sigma_{1}\left(u_{2, n}-u_{2,0}\right)}{n} \sum_{k=1}^{n} \exp \left(u_{1, k-1}\right) \\
& -\rho \sigma_{1} \sum_{k=1}^{n}\left(u_{2, k}-u_{2, k-1}\right) \exp \left(u_{1, k-1}\right) \\
& \zeta_{2}=\sigma_{2} \sum_{k=1}^{n}\left(u_{1, k}-u_{1, k-1}\right) \frac{\exp \left(u_{2, k-1}\right)}{1+\exp \left(u_{2, k-1}\right)} \\
& -\frac{\sigma_{2}\left(u_{2, n}-u_{2,0}\right)}{n} \sum_{k=1}^{n} \frac{\exp \left(u_{2, k-1}\right)}{1+\exp \left(u_{2, k-1}\right)} \\
& +\frac{\rho \sigma_{1}\left(u_{2, n}-u_{2,0}\right)}{n} \sum_{k=1}^{n} \frac{\exp \left(u_{2, k-1}\right)}{1+\exp \left(u_{2, k-1}\right)} \\
& -\rho \sigma_{1} \sum_{k=1}^{n}\left(u_{2, k}-u_{2, k-1}\right) \frac{\exp \left(u_{2, k-1}\right)}{1+\exp \left(u_{2, k-1}\right)}, \\
& \zeta_{3}=\sigma_{1} \sum_{k=1}^{n}\left(u_{2, k}-u_{2, k-1}\right) \frac{\exp \left(u_{1, k-1}\right)}{1+\exp \left(u_{1, k-1}\right)} \\
& -\frac{\sigma_{1}\left(u_{2, n}-u_{2,0}\right)}{n} \sum_{k=1}^{n} \frac{\exp \left(u_{1, k-1}\right)}{1+\exp \left(u_{1, k-1}\right)} \\
& +\frac{\rho \sigma_{2}\left(u_{1, n}-u_{1,0}\right)}{n} \sum_{k=1}^{n} \frac{\exp \left(u_{1, k-1}\right)}{1+\exp \left(u_{1, k-1}\right)} \\
& -\rho \sigma_{2} \sum_{k=1}^{n}\left(u_{1, k}-u_{1, k-1}\right) \frac{\exp \left(u_{1, k-1}\right)}{1+\exp \left(u_{1, k-1}\right)} \\
& \zeta_{4}=\sigma_{1} \sum_{k=1}^{n}\left(u_{2, k}-u_{2, k-1}\right) \exp \left(u_{2, k-1}\right) \\
& -\frac{\sigma_{1}\left(u_{2, n}-u_{2,0}\right)}{n} \sum_{k=1}^{n} \exp \left(u_{2, k-1}\right) \\
& +\frac{\rho \sigma_{2}\left(u_{1, n}-u_{1,0}\right)}{n} \sum_{k=1}^{n} \exp \left(u_{2, k-1}\right) \\
& -\rho \sigma_{2} \sum_{k=1}^{n}\left(u_{1, k}-u_{1, k-1}\right) \exp \left(u_{2, k-1}\right) \text {, }
\end{aligned}
$$

$$
\begin{aligned}
& A_{1}=\frac{1}{\Delta t\left(1-\rho^{2}\right)} \sum_{k=1}^{n}\left[u_{1, k}-u_{1, k-1}-M_{1,2}(k) \Delta t\right]^{2}, \\
& A_{2}=\frac{1}{\Delta t\left(1-\rho^{2}\right)} \sum_{k=1}^{n}\left[u_{2, k}-u_{2, k-1}-M_{2,1}(k) \Delta t\right]^{2}, \\
& B=\frac{\rho}{\Delta t\left(1-\rho^{2}\right)} \sum_{k=1}^{n}\left[u_{1, k}-u_{1, k-1}-M_{1,2}(k) \Delta t\right] \\
& \cdot\left[u_{2, k}-u_{2, k-1}-M_{2,1}(k) \Delta t\right], \\
& M_{1,2}(k)=b_{1}-a_{11} \exp \left(u_{1, k-1}\right)-\frac{c_{2} \exp \left(u_{2, k-1}\right)}{1+\exp \left(u_{2, k-1}\right)}, \\
& M_{2,1}(k)=b_{2}-a_{22} \exp \left(u_{2, k-1}\right)-\frac{c_{1} \exp \left(u_{1, k-1}\right)}{1+\exp \left(u_{1, k-1}\right)} .
\end{aligned}
$$

Then we have the following main result.

Theorem 1. The maximum likelihood estimation of the parameters of system (5) can be expressed as

$$
\begin{aligned}
& \widehat{a_{i i}}=\frac{D_{i i}}{D}, \quad \widehat{c_{i}}=\frac{D_{i}}{D}, \quad(i=1,2), \\
& {\widehat{\sigma_{1}}}^{2}=\frac{A_{1} A_{2}-B \sqrt{A_{1} A_{2}}}{n A_{2}} \text {, } \\
& {\widehat{\sigma_{2}}}^{2}=\frac{A_{1} A_{2}-B \sqrt{A_{1} A_{2}}}{n A_{1}}, \\
& \widehat{b_{1}}=\frac{1}{n \Delta t}\left[u_{1, n}-u_{1,0}+\frac{D_{11}}{D} \Delta t \sum_{k=1}^{n} \exp \left(u_{1, k-1}\right)\right. \\
& \left.+\frac{D_{2}}{D} \Delta t \sum_{k=1}^{n} \frac{\exp \left(u_{2, k-1}\right)}{1+\exp \left(u_{2, k-1}\right)}\right] \text {, } \\
& \widehat{b_{2}}=\frac{1}{n \Delta t}\left[u_{2, n}-u_{2,0}+\frac{D_{22}}{D} \Delta t \sum_{k=1}^{n} \exp \left(u_{2, k-1}\right)\right. \\
& \left.+\frac{D_{1}}{D} \Delta t \sum_{k=1}^{n} \frac{\exp \left(u_{1, k-1}\right)}{1+\exp \left(u_{1, k-1}\right)}\right] \text {, }
\end{aligned}
$$

where

$$
D=\left|\begin{array}{cccc}
\alpha_{1} & \alpha_{2} & \alpha_{3} & \alpha_{4} \\
\beta_{1} & \beta_{2} & \beta_{3} & \beta_{4} \\
\gamma_{1} & \gamma_{2} & \gamma_{3} & \gamma_{4} \\
\eta_{1} & \eta_{2} & \eta_{3} & \eta_{4}
\end{array}\right|, \quad D_{11}=\left|\begin{array}{llll}
\zeta_{1} & \alpha_{2} & \alpha_{3} & \alpha_{4} \\
\zeta_{1} & \beta_{2} & \beta_{3} & \beta_{4} \\
\zeta_{1} & \gamma_{2} & \gamma_{3} & \gamma_{4} \\
\zeta_{1} & \eta_{2} & \eta_{3} & \eta_{4}
\end{array}\right|,
$$$$
D_{22}=\left|\begin{array}{llll}
\alpha_{1} & \alpha_{2} & \alpha_{3} & \zeta_{1} \\
\beta_{1} & \beta_{2} & \beta_{3} & \zeta_{2} \\
\gamma_{1} & \gamma_{2} & \gamma_{3} & \zeta_{3} \\
\eta_{1} & \eta_{2} & \eta_{3} & \zeta_{4}
\end{array}\right|
$$

$$
D_{1}=\left|\begin{array}{llll}
\alpha_{1} & \alpha_{2} & \zeta_{1} & \alpha_{4} \\
\beta_{1} & \beta_{2} & \zeta_{2} & \beta_{4} \\
\gamma_{1} & \gamma_{2} & \zeta_{3} & \gamma_{4} \\
\eta_{1} & \eta_{2} & \zeta_{4} & \eta_{4}
\end{array}\right|, \quad D_{2}=\left|\begin{array}{llll}
\alpha_{1} & \zeta_{1} & \alpha_{3} & \alpha_{4} \\
\beta_{1} & \zeta_{2} & \beta_{3} & \beta_{4} \\
\gamma_{1} & \zeta_{3} & \gamma_{3} & \gamma_{4} \\
\eta_{1} & \zeta_{4} & \eta_{3} & \eta_{4}
\end{array}\right| \text {. }
$$


Proof. Making the change of variable $u_{i, k}=\log x_{i, k},(i=1,2)$, we can rewrite system (5) as

$$
\begin{aligned}
u_{1, k}= & u_{1, k-1}+\left(b_{1}-a_{11} \exp u_{1, k-1}-\frac{c_{2} \exp \left(u_{2, k-1}\right)}{1+\exp \left(u_{2, k-1}\right)}\right) \Delta t \\
& +\sigma_{1} \sqrt{\Delta} t \varepsilon_{1, k}, \\
u_{2, k}= & u_{2, k-1}+\left(b_{2}-a_{22} \exp u_{2, k-1}-\frac{c_{1} \exp \left(u_{1, k-1}\right)}{1+\exp \left(u_{1, k-1}\right)}\right) \Delta t \\
& +\sigma_{1} \sqrt{\Delta} t \varepsilon_{1, k} .
\end{aligned}
$$

Suppose that $\left(U_{1,0}, U_{2,0}\right),\left(U_{1,1}, U_{2,1}\right), \ldots,\left(U_{1, n}, U_{2, n}\right)$ are the observed quantity and $\left(u_{1,0}, u_{2,0}\right),\left(u_{1,1}, u_{2,1}\right), \ldots,\left(u_{1, n}, u_{2, n}\right)$ are the corresponding observed values of system (9). The information flow is given by $\mathscr{F}_{k-1}=\sigma\left(\left(U_{1, l}, U_{2, l}\right), l \leq k-1\right)$. Denote by $\rho$ the correlation coefficient of $U_{1, l}$ and $U_{2, l}$. Then, for given $\mathscr{F}_{k-1}$, the conditional density function of $\left(U_{1, k}, U_{2, k}\right)$ can be expressed as

$$
\begin{array}{r}
f\left(\left(u_{1, k}, u_{2, k}\right) \mid \mathscr{F}_{k-1}\right) \\
=\frac{1}{2 \pi \sigma_{1} \sigma_{2} \Delta t \sqrt{1-\rho^{2}}} \\
\cdot \exp \left\{-\frac{1}{2\left(1-\rho^{2}\right)}\right. \\
\cdot\left[\left(\frac{u_{1, k}-u_{1, k-1}-M_{1,2}(k) \Delta t}{\sigma_{1} \sqrt{\Delta t}}\right)^{2}\right. \\
-2 \rho\left(\frac{u_{1, k}-u_{1, k-1}-M_{1,2}(k) \Delta t}{\sigma_{1} \sqrt{\Delta t}}\right) \\
\cdot\left(\frac{u_{2, k}-u_{2, k-1}-M_{2,1}(k) \Delta t}{\sigma_{2} \sqrt{\Delta t}}\right)^{2} \\
\left.\left.+\left(\frac{u_{2, k}-u_{2, k-1}-M_{2,1}(k) \Delta t}{\sigma_{2} \sqrt{\Delta t}}\right)^{2}\right]\right\},
\end{array}
$$

where

$$
\begin{aligned}
& M_{1,2}(k)=b_{1}-a_{11} \exp \left(u_{1, k-1}\right)-\frac{c_{2} \exp \left(u_{2, k-1}\right)}{1+\exp \left(u_{2, k-1}\right)}, \\
& M_{2,1}(k)=b_{2}-a_{22} \exp \left(u_{2, k-1}\right)-\frac{c_{1} \exp \left(u_{1, k-1}\right)}{1+\exp \left(u_{1, k-1}\right)} .
\end{aligned}
$$

Then, for given $\mathscr{F}_{0}$, the joint conditional density function of $\left(\left(U_{1,1}, U_{2,1}\right),\left(U_{1,2}, U_{2,2}\right), \ldots,\left(U_{1, n}, U_{2, n}\right)\right)$ is

$$
\begin{aligned}
f & \left(\left(\left(u_{1,1}, u_{2,1}\right),\left(u_{1,2}, u_{2,2}\right), \ldots,\left(u_{1, n}, u_{2, n}\right)\right) \mid \mathscr{F}_{0}\right) \\
& =\left(\frac{1}{2 \pi \Delta t \sqrt{1-\rho^{2}} \sigma_{1} \sigma_{2}}\right)^{n}
\end{aligned}
$$

$$
\begin{aligned}
& \cdot \prod_{k=1}^{n} \exp \{- \frac{1}{2\left(1-\rho^{2}\right)} \\
& \cdot\left[\left(\frac{u_{1, k}-u_{1, k-1}-M_{1,2}(k) \Delta t}{\sigma_{1} \sqrt{\Delta t}}\right)^{2}\right. \\
&-2 \rho\left(\frac{u_{1, k}-u_{1, k-1}-M_{1,2}(k) \Delta t}{\sigma_{1} \sqrt{\Delta t}}\right) \\
& \cdot\left(\frac{u_{2, k}-u_{2, k-1}-M_{2,1}(k) \Delta t}{\sigma_{2} \sqrt{\Delta t}}\right) \\
&\left.\left.+\left(\frac{u_{2, k}-u_{2, k-1}-M_{2,1}(k) \Delta t}{\sigma_{2} \sqrt{\Delta t}}\right)^{2}\right]\right\} .
\end{aligned}
$$

With the constants omitted, the logarithmic likelihood function can be written as

$$
\begin{aligned}
& L_{n}\left(b_{1}, b_{2}, a_{11}, a_{22}, c_{1}, c_{2}, \sigma_{1}^{2}, \sigma_{2}^{2}\right) \\
& =-\frac{n}{2}\left(\log \sigma_{1}^{2}+\log \sigma_{2}^{2}\right)-\frac{1}{2 \sigma_{1}^{2} \Delta t\left(1-\rho^{2}\right)} \\
& \quad \cdot \sum_{k=1}^{n}\left[u_{1, k}-u_{1, k-1}-M_{1,2}(k) \Delta t\right]^{2} \\
& \quad+\frac{\rho}{\Delta t\left(1-\rho^{2}\right)} \sum_{k=1}^{n}\left[\frac{u_{1, k}-u_{1, k-1}-M_{1,2}(k) \Delta t}{\sigma_{1}}\right. \\
& \quad \times \frac{1}{2 \sigma_{2}^{2} \Delta t\left(1-\rho^{2}\right)} \sum_{k=1}^{n}\left[u_{2, k}-u_{2, k-1}-M_{2,1}(k) \Delta t\right]^{2} .
\end{aligned}
$$

Taking partial derivative with respect to parameters $b_{1}, b_{2}, a_{11}$, $a_{22}, c_{1}, c_{2}, \sigma_{1}, \sigma_{2}$ in (13), respectively, one obtains the following likelihood equations:

$$
\begin{aligned}
n \rho \Delta t & \sigma_{1} b_{2}-n \sigma_{2} b_{1} \Delta t \\
= & \rho \sigma_{1}\left(u_{2, n}-u_{2,0}\right)-\sigma_{2}\left(u_{1, n}-u_{1,0}\right) \\
& +c_{1} \rho \Delta t \sigma_{1} \sum_{k=1}^{n} \frac{\exp \left(u_{1, k}\right)}{1+\exp \left(u_{1, k}\right)} \\
& -c_{2} \Delta t \sigma_{2} \sum_{k=1}^{n} \frac{\exp \left(u_{2, k}\right)}{1+\exp \left(u_{2, k}\right)}+a_{22} \rho \Delta t \sigma_{1} \sum_{k=1}^{n} \exp \left(u_{2, k-1}\right) \\
& -a_{11} \Delta t \sigma_{2} \sum_{k=1}^{n} \exp \left(u_{1, k-1}\right),
\end{aligned}
$$




$$
\begin{aligned}
n \rho \Delta t & \left(\sigma_{2} b_{1}-\sigma_{1} b_{2}\right) \\
= & \rho \sigma_{2}\left(u_{1, n}-u_{1,0}\right)-\sigma_{1}\left(u_{2, n}-u_{2,0}\right) \\
& +c_{2} \rho \Delta t \sigma_{2} \sum_{k=1}^{n} \frac{\exp \left(u_{2, k}\right)}{1+\exp \left(u_{2, k}\right)} \\
& -c_{1} \Delta t \sigma_{1} \sum_{k=1}^{n} \frac{\exp \left(u_{1, k}\right)}{1+\exp \left(u_{1, k}\right)}+a_{11} \rho \Delta t \sigma_{2} \sum_{k=1}^{n} \exp \left(u_{1, k-1}\right) \\
& -a_{22} \Delta t \sigma_{1} \sum_{k=1}^{n} \exp \left(u_{2, k-1}\right),
\end{aligned}
$$$$
\sigma_{2} \sum_{k=1}^{n}\left[u_{1, k}-u_{1, k-1}-M_{1,2}(k) \Delta t\right] \exp \left(u_{1, k-1}\right)
$$$$
=\rho \sigma_{1} \sum_{k=1}^{n}\left[u_{2, k}-u_{2, k-1}-M_{2,1}(k) \Delta t\right] \exp \left(u_{1, k-1}\right),
$$$$
\sigma_{2} \sum_{k=1}^{n}\left[u_{1, k}-u_{1, k-1}-M_{1,2}(k) \Delta t\right] \frac{\exp \left(u_{2, k-1}\right)}{1+\exp \left(u_{2, k-1}\right)}
$$$$
=\rho \sigma_{1} \sum_{k=1}^{n}\left[u_{2, k}-u_{2, k-1}-M_{2,1}(k) \Delta t\right] \frac{\exp \left(u_{2, k-1}\right)}{1+\exp \left(u_{2, k-1}\right)},
$$$$
\sigma_{1} \sum_{k=1}^{n}\left[u_{2, k}-u_{2, k-1}-M_{2,1}(k) \Delta t\right] \frac{\exp \left(u_{1, k-1}\right)}{1+\exp \left(u_{1, k-1}\right)}
$$$$
=\rho \sigma_{2} \sum_{k=1}^{n}\left[u_{1, k}-u_{1, k-1}-M_{1,2}(k) \Delta t\right] \frac{\exp \left(u_{1, k-1}\right)}{1+\exp \left(u_{1, k-1}\right)},
$$$$
\sigma_{1} \sum_{k=1}^{n}\left[u_{2, k}-u_{2, k-1}-M_{2,1}(k) \Delta t\right] \exp \left(u_{2, k-1}\right)
$$$$
=\rho \sigma_{2} \sum_{k=1}^{n}\left[u_{1, k}-u_{1, k-1}-M_{1,2}(k) \Delta t\right] \exp \left(u_{2, k-1}\right),
$$$$
-\frac{n}{2 \sigma_{1}^{2}}+\frac{1}{2 \sigma_{1}^{4} \Delta t\left(1-\rho^{2}\right)} \sum_{k=1}^{n}\left[u_{1, k}-u_{1, k-1}-M_{1,2}(k) \Delta t\right]^{2}
$$$$
=\frac{\rho}{2 \sigma_{1}^{3} \sigma_{2} \Delta t\left(1-\rho^{2}\right)} \sum_{k=1}^{n}\left[u_{1, k}-u_{1, k-1}-M_{1,2}(k) \Delta t\right]
$$$$
\cdot\left[u_{2, k}-u_{2, k-1}-M_{2,1}(k) \Delta t\right],
$$

$$
\begin{gathered}
-\frac{n}{2 \sigma_{2}^{2}}+\frac{1}{2 \sigma_{2}^{4} \Delta t\left(1-\rho^{2}\right)} \sum_{k=1}^{n}\left[u_{2, k}-u_{2, k-1}-M_{2,1}(k) \Delta t\right]^{2} \\
=\frac{\rho}{2 \sigma_{1} \sigma_{2}^{3} \Delta t\left(1-\rho^{2}\right)} \sum_{k=1}^{n}\left[u_{1, k}-u_{1, k-1}-M_{1,2}(k) \Delta t\right] \\
\cdot\left[u_{2, k}-u_{2, k-1}-M_{2,1}(k) \Delta t\right] .
\end{gathered}
$$

By (14) and (15), one has

$$
\begin{gathered}
b_{1}=\frac{1}{n \Delta t}\left[u_{1, n}-u_{1,0}+a_{11} \Delta t \sum_{k=1}^{n} \exp \left(u_{1, k-1}\right)\right. \\
\left.+c_{2} \Delta t \sum_{k=1}^{n} \frac{\exp \left(u_{2, k-1}\right)}{1+\exp \left(u_{2, k-1}\right)}\right], \\
b_{2}=\frac{1}{n \Delta t}\left[u_{2, n}-u_{2,0}+a_{22} \Delta t \sum_{k=1}^{n} \exp \left(u_{2, k-1}\right)\right. \\
\left.+c_{1} \Delta t \sum_{k=1}^{n} \frac{\exp \left(u_{1, k-1}\right)}{1+\exp \left(u_{1, k-1}\right)}\right] .
\end{gathered}
$$

It then follows from (22), (23), and (16)-(19) that

$$
\begin{aligned}
& \alpha_{1} a_{11}+\alpha_{2} c_{2}+\alpha_{3} c_{1}+\alpha_{4} a_{22} \\
&= \sigma_{2} \sum_{k=1}^{n}\left(u_{1, k}-u_{1, k-1}\right) \exp \left(u_{1, k-1}\right) \\
&-\frac{\sigma_{2}\left(u_{1, n}-u_{1,0}\right)}{n} \sum_{k=1}^{n} \exp \left(u_{1, k-1}\right) \\
&+\frac{\rho \sigma_{1}\left(u_{1, n}-u_{1,0}\right)}{n} \sum_{k=1}^{n} \exp \left(u_{1, k-1}\right) \\
&-\rho \sigma_{1} \sum_{k=1}^{n}\left(u_{2, k}-u_{2, k-1}\right) \exp \left(u_{1, k-1}\right), \\
& \beta_{1} a_{11}+\beta_{2} c_{2}+\beta_{3} c_{1}+\beta_{4} a_{22} \\
&=\sigma_{2} \sum_{k=1}^{n}\left(u_{1, k}-u_{1, k-1}\right) \frac{\exp \left(u_{2, k-1}\right)}{1+\exp \left(u_{2, k-1}\right)} \\
& \quad-\frac{\sigma_{2}\left(u_{2, n}-u_{2,0}\right)}{n} \sum_{k=1}^{n} \frac{\exp \left(u_{2, k-1}\right)}{1+\exp \left(u_{2, k-1}\right)} \\
&+\frac{\rho \sigma_{1}\left(u_{2, n}-u_{2,0}\right)}{n} \sum_{k=1}^{n} \frac{\exp \left(u_{2, k-1}\right)}{1+\exp \left(u_{2, k-1}\right)} \\
& \quad+\frac{\rho \sigma_{2}\left(u_{1, n}-u_{1,0}\right)}{n} \sum_{k=1}^{n} \frac{\exp \left(u_{1, k-1}\right)}{1+\exp \left(u_{1, k-1}\right)} \\
& \gamma_{1} a_{11}+\gamma_{2} \mathcal{c}_{2}+\gamma_{3} c_{1}+\gamma_{4} a_{22} \\
&=\sigma_{1} \sum_{k=1}^{n}\left(u_{2, k}-u_{2, k-1}\right) \frac{\exp \left(u_{1, k-1}\right)}{1+\exp \left(u_{1, k-1}\right)}, \\
& 1+\exp \left(u_{1, k-1}\right) \\
& \sigma_{k=1}^{n}\left(u_{2, k}-u_{2, k-1}\right) \frac{\exp \left(u_{2, k-1}\right)}{1+\exp \left(u_{2, k-1}\right)}, \\
& 1+\exp \left(u_{1, k-1}\right)
\end{aligned}
$$




$$
\begin{aligned}
\eta_{1} a_{11}+\eta_{2} c_{2}+\eta_{3} c_{1}+\eta_{4} a_{22} \\
=\sigma_{1} \sum_{k=1}^{n}\left(u_{2, k}-u_{2, k-1}\right) \exp \left(u_{2, k-1}\right) \\
\quad-\frac{\sigma_{1}\left(u_{2, n}-u_{2,0}\right)}{n} \sum_{k=1}^{n} \exp \left(u_{2, k-1}\right) \\
+\frac{\rho \sigma_{2}\left(u_{1, n}-u_{1,0}\right)}{n} \sum_{k=1}^{n} \exp \left(u_{2, k-1}\right) \\
\quad-\rho \sigma_{2} \sum_{k=1}^{n}\left(u_{1, k}-u_{1, k-1}\right) \exp \left(u_{2, k-1}\right) .
\end{aligned}
$$

So, the Gramer rule implies that

$$
\widehat{a_{i i}}=\frac{D_{i i}}{D}, \quad \widehat{c_{i}}=\frac{D_{i}}{D}, \quad(i=1,2) .
$$

Further, from (22) and (23), one achieves the fact that

$$
\begin{gathered}
\widehat{b_{1}}=\frac{1}{n \Delta t}\left[u_{1, n}-u_{1,0}+\frac{D_{11}}{D} \Delta t \sum_{k=1}^{n} \exp \left(u_{1, k-1}\right)\right. \\
\left.+\frac{D_{2}}{D} \Delta t \sum_{k=1}^{n} \frac{\exp \left(u_{2, k-1}\right)}{1+\exp \left(u_{2, k-1}\right)}\right], \\
\widehat{b_{2}}=\frac{1}{n \Delta t}\left[\begin{array}{c}
u_{2, n}-u_{2,0}+\frac{D_{22}}{D} \Delta t \sum_{k=1}^{n} \exp \left(u_{2, k-1}\right) \\
\left.+\frac{D_{1}}{D} \Delta t \sum_{k=1}^{n} \frac{\exp \left(u_{1, k-1}\right)}{1+\exp \left(u_{1, k-1}\right)}\right] .
\end{array}\right.
\end{gathered}
$$

It is easy to see from (20), (21), (23), and (24) that

$$
\begin{aligned}
& -\frac{n}{\sigma_{1}^{2}}+\frac{A_{1}}{\sigma_{1}^{4}}-\frac{B}{\sigma_{1}^{3} \sigma_{2}}=0, \\
& -\frac{n}{\sigma_{2}^{2}}+\frac{A_{2}}{\sigma_{2}^{4}}-\frac{B}{\sigma_{2}^{3} \sigma_{1}}=0 .
\end{aligned}
$$

Then one achieves the fact that

$$
\begin{aligned}
& {\widehat{\sigma_{1}}}^{2}=\frac{A_{1} A_{2}-B \sqrt{A_{1} A_{2}}}{n A_{2}}, \\
& {\widehat{\sigma_{2}}}^{2}=\frac{A_{1} A_{2}-B \sqrt{A_{1} A_{2}}}{n A_{1}} .
\end{aligned}
$$

This completes the proof.

\section{Numerical Simulations}

In this section, we give some simulation results of the above estimators to compare the true values and the estimators. In Tables 1-4, we fix the true values of parameters as follows: $a_{11}=2, a_{22}=1, b_{1}=1, b_{2}=1.5, c_{1}=1.2, c_{2}=1.8$, $\rho=0.65, \sigma_{1}^{2}=0.09$, and $\sigma_{2}^{2}=0.16$, the number of the sample
TABLE 1: The simulation values of the MLE of $\left(a_{11}, a_{22}\right)$ when $\Delta t=$ 0.1 .

\begin{tabular}{lccccc}
\hline True & Size & \multicolumn{2}{c}{ Average } & \multicolumn{2}{c}{ AE } \\
$\left(a_{11}, a_{22}\right)$ & $n$ & $a_{11}$-MLE & $a_{22}$-MLE & $a_{11}$ & $a_{22}$ \\
\hline \multirow{3}{*}{$(2,1)$} & 500 & 1.991630 & 1.014662 & 0.008370 & 0.014662 \\
& 1000 & 2.004029 & 0.992371 & 0.004029 & 0.007629 \\
& 2000 & 2.006013 & 1.005748 & 0.006013 & 0.005748 \\
\hline
\end{tabular}

TABLE 2: The simulation values of the MLE of $\left(b_{1}, b_{2}\right)$ when $\Delta t=0.1$.

\begin{tabular}{lccccc}
\hline True & Size & \multicolumn{2}{c}{ Average } & \multicolumn{2}{c}{ AE } \\
$\left(b_{1}, b_{2}\right)$ & $n$ & $b_{1}$-MLE & $b_{2}$-MLE & $b_{1}$ & $b_{2}$ \\
\hline \multirow{4}{*}{$(1,1.5)$} & 500 & 1.013570 & 1.508662 & 0.013570 & 0.008662 \\
& 1000 & 0.993755 & 1.504625 & 0.006245 & 0.004625 \\
& 2000 & 1.004742 & 1.496252 & 0.004742 & 0.003748 \\
\hline
\end{tabular}

TABLE 3: The simulation values of the MLE of $\left(c_{1}, c_{2}\right)$ when $\Delta t=0.1$.

\begin{tabular}{lccccc}
\hline True & Size & \multicolumn{2}{c}{ Average } & \multicolumn{2}{c}{ AE } \\
$\left(c_{1}, c_{2}\right)$ & $n$ & $c_{1}$-MLE & $c_{2}$-MLE & $c_{1}$ & $c_{2}$ \\
\hline & 500 & 1.225380 & 1.785378 & 0.025380 & 0.014622 \\
$(1.2,1.8)$ & 1000 & 1.209025 & 1.808455 & 0.009025 & 0.008455 \\
& 2000 & 1.207742 & 1.854748 & 0.007742 & 0.054748 \\
\hline
\end{tabular}

TABLE 4: The simulation values of the $\operatorname{MLE}$ of $\left(\sigma_{1}^{2}, \sigma_{2}^{2}\right)$ when $\Delta t=0.1$.

\begin{tabular}{lccccc}
\hline True & Size & \multicolumn{2}{c}{ Average } & \multicolumn{2}{c}{ AE } \\
$\left(\sigma_{1}^{2}, \sigma_{2}^{2}\right)$ & $n$ & $\sigma_{1}^{2}$-MLE & $\sigma_{2}^{2}$-MLE & $\sigma_{1}^{2}$ & $\sigma_{2}^{2}$ \\
\hline \multirow{3}{*}{$(0.09,0.16)$} & 500 & 0.093120 & 0.164635 & 0.003120 & 0.004635 \\
& 1000 & 0.086975 & 0.163322 & 0.003025 & 0.003322 \\
& 2000 & 0.093742 & 0.163342 & 0.003742 & 0.003342 \\
\hline
\end{tabular}

"size $n$ " increases from 500 to 2000, the data of the columns named Average are obtained by the average of 10 MLEs from the data coming from (5) with $x_{1}(0)=0.5, x_{2}(0)=0.44$, $a_{11}=2, a_{22}=1, b_{1}=1, b_{2}=1.5, c_{1}=1.2, c_{2}=1.8$, $\rho=0.65, \sigma_{1}^{2}=0.09, \sigma_{2}^{2}=0.16$, and $\Delta t=0.1$. The columns named AE show the absolute error of MLE. In Tables 1-4, it is easy to see that there is a correlation between the absolute error of MLE and the sample size. Generally speaking, with the increase in the number of the sample, the absolute error of MLE will decrease. Therefore, it is reasonable to estimate the parameters of system (5) by MLE.

\section{Conflict of Interests}

The authors declare that they have no conflict of interests. 


\section{Acknowledgments}

The work is supported by the National Natural Science Foundation of China (no. 11261017), the Key Laboratory of Biological Resources Protection and Utilization of Hubei Province (PKLHB1329, PKLHB1327), and the Key Subject of Hubei Province (Mathematics).

\section{References}

[1] R. P. Agarwal, Difference Equations and Inequalities: Theory, Methods and Applications, Monographs and Textbooks in Pure and Applied Mathematics, vol. 228, Marcel Dekker, New York, NY, USA, 2000.

[2] Z. Liu and L. Chen, "Positive periodic solution of a general discrete non-autonomous difference system of plankton allelopathy with delays," Journal of Computational and Applied Mathematics, vol. 197, no. 2, pp. 446-456, 2006.

[3] C. Niu and X. Chen, "Almost periodic sequence solutions of a discrete Lotka-Volterra competitive system with feedback control," Nonlinear Analysis: Real World Applications, vol. 10, no. 5, pp. 3152-3161, 2009.

[4] W. J. Qin, Z. J. Liu, and Y. P. Chen, "Permanence and global stability of positive periodic solutions of a discrete competitive system," Discrete Dynamics in Nature and Society, vol. 2009, Article ID 830537, 13 pages, 2009.

[5] Z. Li, F. Chen, and M. He, "Almost periodic solutions of a discrete Lotka-Volterra competition system with delays," Nonlinear Analysis: Real World Applications, vol. 12, no. 4, pp. 2344-2355, 2011.

[6] Q. L. Wang and Z. J. Liu, "Uniformly asymptotic stability of almost periodic solutions for a delay difference system of plankton allelopathy," Advances in Difference Equations, vol. 2013, article 283, 2013.

[7] R. M. May, Stability and Complexity in Model Ecosystems, Princeton University Press, New York, NY, USA, 2001.

[8] D.-Q. Jiang, B.-X. Zhang, D.-H. Wang, and N.-Z. Shi, "Existence, uniqueness, and global attractivity of positive solutions and MLE of the parameters to the logistic equation with random perturbation," Science in China Series A: Mathematics, vol. 50, no. 7, pp. 977-986, 2007.

[9] Z. W. Liu, Estimation of parameters and asymptotic charaacteristic of discrete stochastic Lotka-Volterra competition system [M.S. thesis], Northeast Normal University, Changchun, China, 2006.

[10] H. Xiang and Z. Liu, "The optimal control and MLE of parameters of a stochastic single-species system," Discrete Dynamics in Nature and Society, vol. 2012, Article ID 676871, 12 pages, 2012.

[11] K. Gopalsamy, Stability and Oscillations in Delay Differential Equations of Population Dynamics, Kluwer Academic Publishers, Dordrecht, The Netherlands, 1992. 


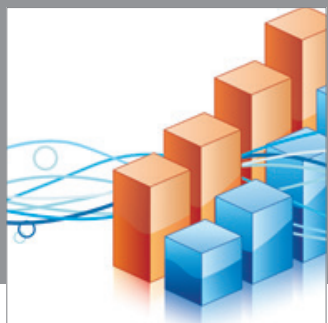

Advances in

Operations Research

mansans

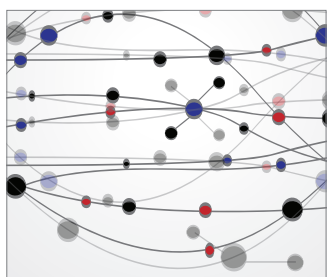

The Scientific World Journal
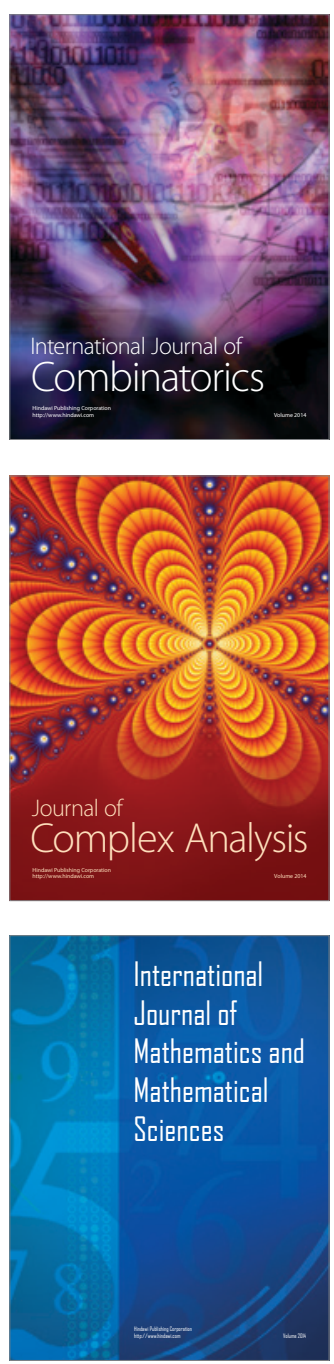
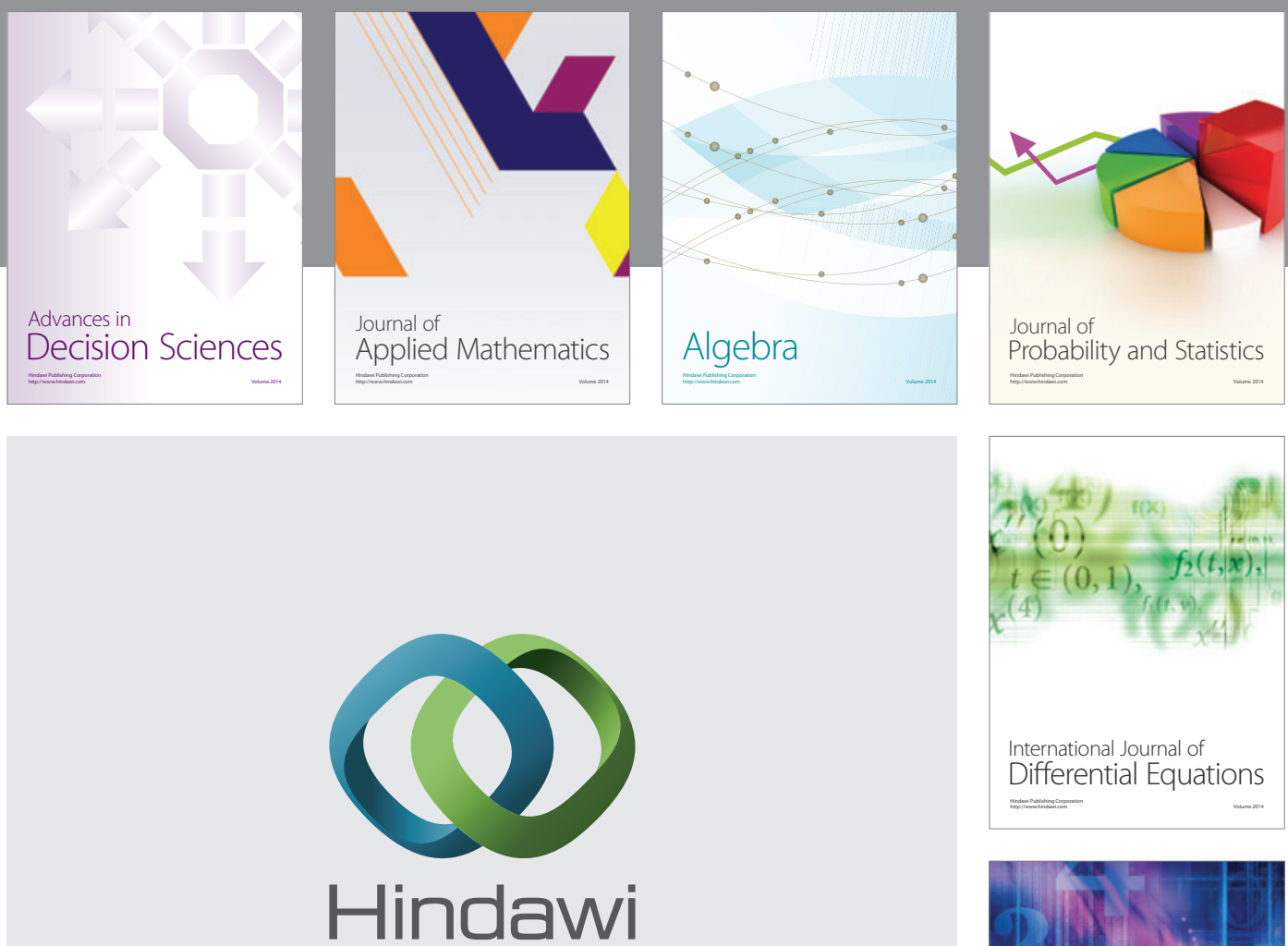

Submit your manuscripts at http://www.hindawi.com
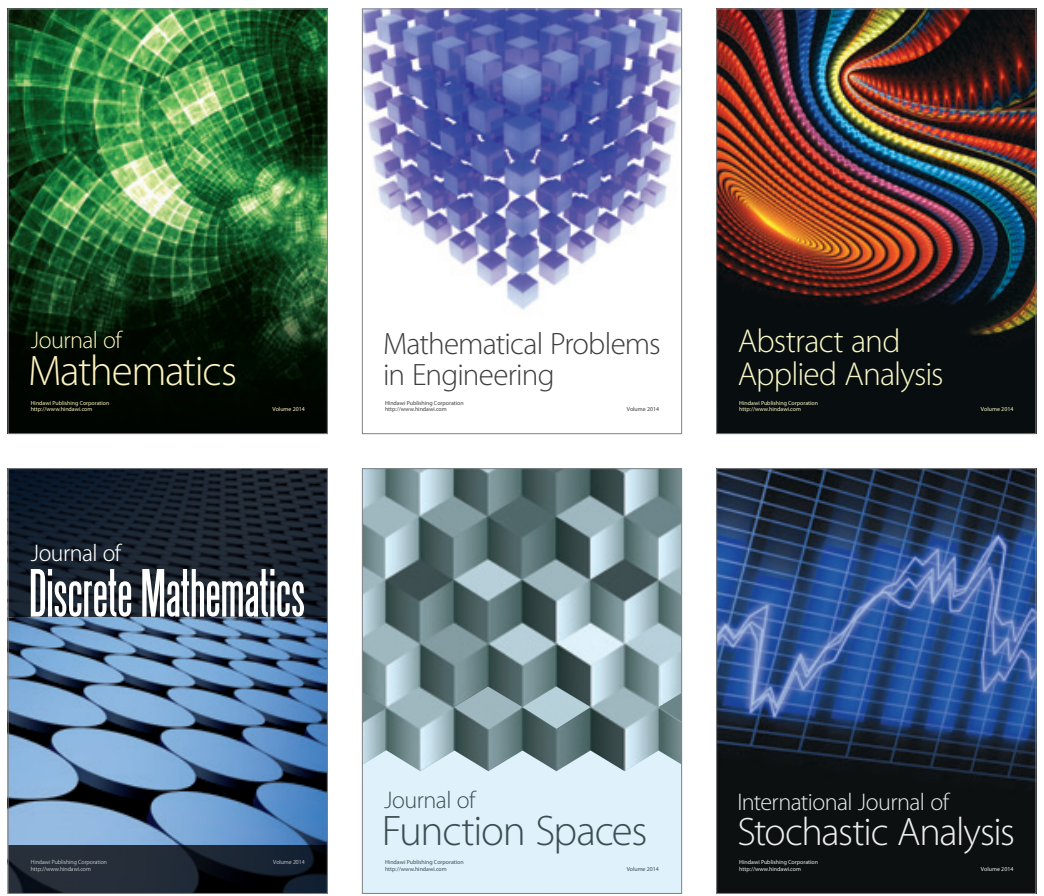

Journal of

Function Spaces

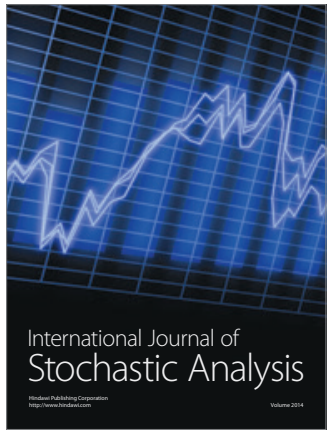

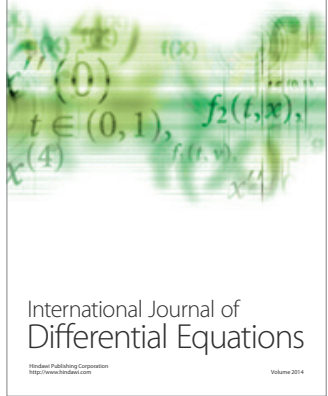
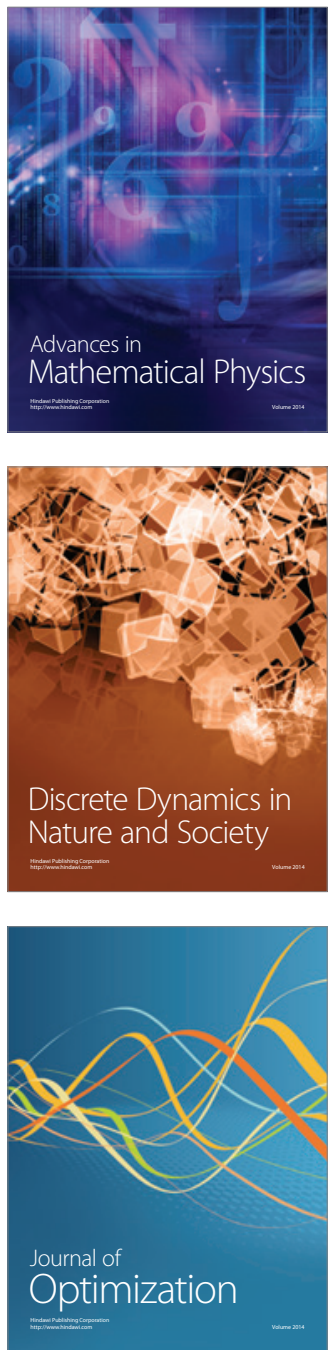